\title{
Robust Sparse Network Equivalent for Large Systems: Part I-Methodology
}

\author{
Wallace do Couto Boaventura, Member, IEEE, Adam Semlyen, Life Fellow, IEEE, M. Reza Iravani, Fellow, IEEE, \\ and Amauri Lopes, Member, IEEE
}

\begin{abstract}
This paper deals with the calculation of a sparse network equivalent (SNE) for the analysis of electromagnetic transients in large systems. The main feature of the new approach is the enforcement of sparsity, stability, passivity, and accuracy at specific frequencies of the equivalent. The procedure is based on time-domain fitting with quadratic programming to enforce the constraints. The SNE can be interfaced with transient calculation programs directly in time domain. Results are presented in the companion paper.
\end{abstract}

Index Terms-Discrete time, electromagnetic transients, network equivalent, quadratic programming, time-domain fitting.

\section{INTRODUCTION}

$\mathbf{T}$ RANSIENT studies play an important role in the design and operation of an electric power system. In such studies, there is usually a study zone, a restricted portion of the system, and an external system, which comprises the rest of the system. If a complete representation is adopted for the external system, the computational effort required for the calculations may be excessive. This is particularly true for the case of large systems especially when frequency dependent models are used for transmission lines. Even if only a part of the external system is to be used in the calculation, it is difficult to establish which part should be neglected. Network equivalents are used in this situation to reduce the computational burden for the transient calculation of the whole system by providing a suitable representation for the external system. Several contributions about calculation of network equivalents using either frequency-domain or time-domain fitting techniques are reported in [1]-[5] and [6]-[11], respectively. Most methodologies based on frequency-domain fitting, including [1]-[4], produce reduced-order models. In these, only the main characteristics of the external system (namely the largest peaks in the frequency response) are modeled. Reference [5] does not use any approximation in modeling the frequency response of the external system. A special procedure involving fast Fourier transform (FFT) is used for the frequency response in its original form. Our motivation to use time-domain instead of

Manuscript received January 16, 2003. This work was supported in part by the Natural Sciences and Engineering Research Council of Canada, and in part by CAPES, Brazil.

W. C. Boanaventura is with the Department of Electrical and Computer Engineering, University of Toronto, Toronto, ON M5S 3G4, Canada, on leave from the Department of Electrical Engineering, Federal University of Minas Gerais, Belo Horizonte, Brazil (e-mail: wventura@cpdee.ufmg.br).

A. Semlyen and M. R. Iravani are with the Department of Electrical and Computer Engineering, University of Toronto, Toronto, ON M5S 3G4, Canada (e-mail: adam.semlyen@utoronto.ca; iravani@ecf.utoronto.ca).

A. Lopes is with the Department of Communications, University of Campinas, Campinas 13.083-970, Brazil (e-mail: amauri@ decom.fee.unicamp.br).

Digital Object Identifier 10.1109/TPWRS.2003.818603 frequency-domain fitting comes from the possibility of deriving full-order models with reduced computational burden, which is permitted by the sparsity. This issue is further discussed in Section II of the companion paper. Regarding time-domain fitting methodologies, we follow in general terms [6]-[11], but we deal explicitly with sparsity and robustness in the network equivalent calculation.

This paper presents a methodology for deriving a sparse network equivalent (SNE) based on time-domain fitting procedures. The main feature of the new procedure is the embedded enforcement of robustness and sparsity in the calculation of the equivalent. Robustness implies both stability and passivity and sparsity increases the computational efficiency. Also enforced is the accuracy of the equivalent at specific important frequencies like 0 and $60 \mathrm{~Hz}$. As it is derived in discrete-time domain, the SNE can be directly interfaced with the study zone for the simulation of electromagnetic transients. The computational efficiency of the equivalent is ensured by its sparsity. The results presented in the companion paper demonstrate the feasibility of the methodology in deriving equivalents for systems composed of up to 100 transmission lines. Computational efficiency and accuracy in the context of digital electromagnetic transient calculations have been examined in [12]-[20].

In the remainder of this paper, the methodology for the calculation of the equivalent is described. Section II presents the general formulation for the methodology followed by the fitting and constraint equations in Sections III and IV. Details of implementation are given in Section V and conclusions in Section VI.

\section{OUtLINE OF THE METHODOLOGY}

The data required for computing the equivalent are obtained either from digital time-domain simulation of the external system or from measurements. For the latter, the errors due to digitization and noise can be significant but the methods to deal with this issue are not addressed in this paper. Furthermore, the difficulties in having a large system in its entirety available for measurements pose additional constraints in the use of this kind of data. During the commissioning of new lines, it may be possible to take measurements in a small part of the system. Fitting procedures usually require a solution of an overdetermined set of linear equations. This, however, does not ensure stability, passivity, sparsity, and accuracy (at specific frequencies) of the equivalent. Therefore, in this paper, a set of linear constraints is added to the set of linear equations related to the fitting. In a general formulation we solve

$$
\min _{x}\|A x-c\| \quad D x \leq e
$$


where $A$ and $c$ correspond to the fitting equations and $D$ and $e$ to the constraint equations, to be detailed later. A solution for $x$ is obtained when the fitting equations are overdetermined by using quadratic programming [21], [25].

The fitting equations, presented in the next section, are deduced from the discrete nature of the equivalent and enforce its sparsity. In Section IV, constraint equations for stability, passivity, and accuracy at specific frequencies are derived and combined to obtain $D$ and $e$. For multiphase systems, the equivalents are calculated treating each mode separately via modal decomposition techniques.

\section{FITTING EQUATIONS}

A single port equivalent for an external system is derived in this section. Assuming time invariance and zero initial conditions, a linear system, seen as a single port network, can be fully characterized in discrete-time domain by a linear constant-coefficient equation [22]. Two data sequences-a voltage/current pair $v(n)$ and $i(n)$, considered as input and output, respectively, are used to calculate the equivalent. These data sequences of length $N$ are taken at the port of the external system. The data are assumed accurate, disregarding errors due to discretization which are inherent in digital simulations. The difference equation of order $p$ (referring to the output) relates $v(n)$ and $i(n)$ and represents the equivalent as

$$
\sum_{k=0}^{p} a_{k} i(n-k)=\sum_{k=0}^{q} b_{k} v(n-k) \quad(n=0, \ldots, N-1) .
$$

The coefficients $a_{k}$ and $b_{k}$ in (3), which describe the equivalent, are to be determined. $q$ is the number of past terms related to the input and used to compute the present value of the output.

The input $v(n)$ must be such that the calculated coefficients $a_{k}$ and $b_{k}$ can characterize the external system in the desired frequency range (i.e., from zero to the maximum frequency allowed by the time step). The choice of the time step is ruled by the purpose of the transient simulation in which the equivalent is to be used. A unit-step input voltage is generally used for $v(n)$ as it can be easily represented in transient calculation programs and meets the frequency requirements. This input signal excites the external system in a broad range of frequencies, allowing thus its proper characterization. The coefficient $a_{0}$ in (2) can be set to 1 without loss of generality. Setting $q=p$ (see Section III-A below) and rearranging the terms in (2), we build a set of $N$ linear equations, assuming $N>2 p+1$. This results in (3), or (4), where the subscripts of the variables $v$ and $i$ indicate the corresponding time index in the sequences $v(n)$ and $i(n)$

$$
\begin{aligned}
& {\left[\begin{array}{cccc|cccc}
0 & 0 & \cdot & 0 & -v_{0} & 0 & \cdot & 0 \\
i_{0} & 0 & \cdot & 0 & -v_{1} & -v_{0} & \cdot & 0 \\
\cdot & \cdot & \cdot & 0 & \cdot & \cdot & \cdot & 0 \\
i_{p-2} & i_{p-3} & \cdot & 0 & -v_{p-1} & -v_{p-2} & \cdot & 0 \\
\hline i_{p-1} & i_{p-2} & \cdot & i_{0} & -v_{p} & -v_{p-1} & \cdot & -v_{0} \\
\cdot \cdot & \cdot & \cdot & \cdot & \cdot & \cdot & \cdot & \cdot \\
i_{N-2} & i_{N-3} & \cdot & i_{N-p-1} & -v_{N-1} & -v_{N-2} & \cdot & -v_{N-p-1}
\end{array}\right] } \\
& \times\left[\begin{array}{c}
a_{1} \\
\cdot \\
a_{p} \\
b_{0} \\
\cdot \\
\cdot \\
b_{p}
\end{array}\right]=\left[\begin{array}{c}
-i_{0} \\
-i_{1} \\
\cdot \\
-i_{p-1} \\
-i_{p} \\
\cdot \\
-i_{N-1}
\end{array}\right]
\end{aligned}
$$

or

$$
\left[\begin{array}{ll}
C & -V
\end{array}\right]\left[\begin{array}{l}
a \\
b
\end{array}\right]=[-i] .
$$

Note that if a unit-impulse voltage is used for $v(n)$, the first $p+1$ rows of submatrix $V$ are reduced to the identity matrix. The coefficients $b_{k}$ would then be just a linear combination of the coefficients $a_{k}$ as in Prony's work [23]. This permits a separate solution for the unknowns $a_{k}$ and $b_{k}$, reducing the size of the matrices and the corresponding computational effort. However, the constraints of stability, passivity, and accuracy at specific frequencies depend on both $a_{k}$ and $b_{k}$. Therefore, we use a step response for simultaneous calculation of $a_{k}$ and $b_{k}$. This also makes the extension of the procedure easier when the data for $v(n)$ and $i(n)$ are obtained from measurements.

$N$ is larger then $2 p+1$ if (3) is overdetermined. Our studies indicate that a redundancy of about two results in a fairly accurate solution. The order $p$ and the modification of (3) to enforce sparsity are discussed in the following sections.

Rearranging the terms in (2) and substituting $q=p$ and $a_{0}=$ 1 , we get the normalized equation

$$
i(n)=b_{0} v(n)+\sum_{k=1}^{p}\left(b_{k} v(n-k)-a_{k} i(n-k)\right) .
$$

The coefficient $b_{0}$ in (5) has the dimension of an admittance and the summation is computed only from past values. This allows the integration of the equivalent into a transient calculation program. When dealing with multiphase systems, the equivalents for the line and ground modes (based on modal transformation matrices) are used to obtain the end results in phase domain.

\section{A. Determination of the Order of the Equivalent}

Each transmission line in the external system requires an "infinite-order" model (with an infinitesimal time step) for exact representation. For transient simulations, sufficiently accurate results are achieved using a reasonably small time step. There is an intrinsical relation between the time step, the "length" of the external system, and the order of the equivalent. Reducing the external system to a single, open-ended, transmission line and analyzing the current wave reflections due to energization by a unit step voltage, we obtain information about the order of the equivalent. The first reflected traveling wave arrives at the sending end bus from the far end at a time corresponding to twice the line travel time. Thus, an equivalent must have an order equal to this time divided by the time step. Also from this analysis, $q$ in (2) must be equal to $p$ to correctly account for multiple reflections. When the external system is composed of several transmission lines, the current-wave reflections due to all connections and terminations contribute to the response characteristic. It is thus difficult to assign a proper order for the equivalent only based on the configuration and length of the transmission lines of the external system.

To determine $p$, we define two limits for the "length" (in time steps) of the external system, which are usually the limits for $p$. The minimum length of a network is defined as the double of the time required for a signal to arrive from the sending bus to the farthest node in the network, through the shortest path, divided 
by the time step. By computing the double of all transmission line travel times divided by the time step, we get the maximum length of a network. A radial network has $p$ approaching the minimum length while a meshed network has $p$ close to the maximum length. This analysis suggests that $p$ increases indefinitely with the length of the network. However, our studies indicate that even a very large network can be accurately approximated by an equivalent of a comparatively small $p$. We attribute this to the presence of losses in the network and to its length. Losses make the traveling waves arriving from the farthest nodes relatively small in amplitude so that their influence is barely noticed in the waveforms taken at the sending bus. We can also establish a relation between the order for the line-mode and ground-mode equivalents. The ground mode has a lower velocity of propagation and correspondingly results in a larger "length" for the network. Accordingly, we use a larger order for the ground-mode equivalent compared to the line-mode equivalent. To further estimate the value of $p$, we perform a numerical rank analysis of the matrices of (3), as described below.

The data corresponding to $v(n)$ or $i(n)$, respectively, used to build $C$ or $V$ in (4), is corrupted by round-off noise due to the limited number of significant digits used in transient calculations. Although the noise is very low, its effect is easily noticed using singular value decomposition (SVD) [24], [25] of the matrices of (3). As shown by the dashed lines, we have four submatrices in (3): $C_{U}, V_{U}$ and $C_{L}, V_{L}$ (for upper and lower partitions respectively) and all correspond to matrix $A$ of (1). When solving an ordinary set of linear equations, one would evaluate the rank of $A$. Due to the structure of $C$ and $V$, we only use the lower partition $C_{L}$ in this analysis. Partitions $C_{U}$ and $V_{U}$ contribute to the rank of $A$ with $p$ linearly independent rows, regardless of how large $p$ is. This is due to the fact that the upper triangles are filled with zeros (accounting for the initial conditions). If $p$ is overestimated, the upper partitions lead to erroneous information about the intrinsical order of the system. $C_{L}$, not $V_{L}$, is used because the sequences $v(n)$ and $i(n)$ are produced using a voltage source. Building $C_{L}$ for a value of $p$ much larger than the presumed order of the external system and calculating its SVD, one can notice that several singular values are negligible. These account for the round-off noise present in $C_{L}$. Thus, a suitable value for $p$ is the index of the lowest significant singular value (i.e. the numerical rank of $C_{L}$ ). This choice for the value of $p$ usually results in the well-conditioning of (3), allowing proper calculation of the $2 p+1$ unknowns, the coefficients sets $a_{k}$ and $b_{k}$.

Due to the high losses inherent in the ground mode, the traveling waves corresponding to the reflections at the farthest nodes are highly attenuated. This is reflected in the SVD analysis of $C_{L}$ for the ground mode as a gradual decrease in its singular values. The SVD-based procedure for the determination of $p$ would indicate a value much smaller than a suitable one in this case. As the losses are low in the line mode, the procedure reliably results in an appropriate value of $p$ for this mode. Thus, $p$ for the ground mode is determined by multiplying the corresponding value for the line mode by a factor greater than one which accounts for the differences in the travel times of the modes. From several network equivalent calculations, we have found that a suitable range for the multiplying factor is 1.1 to

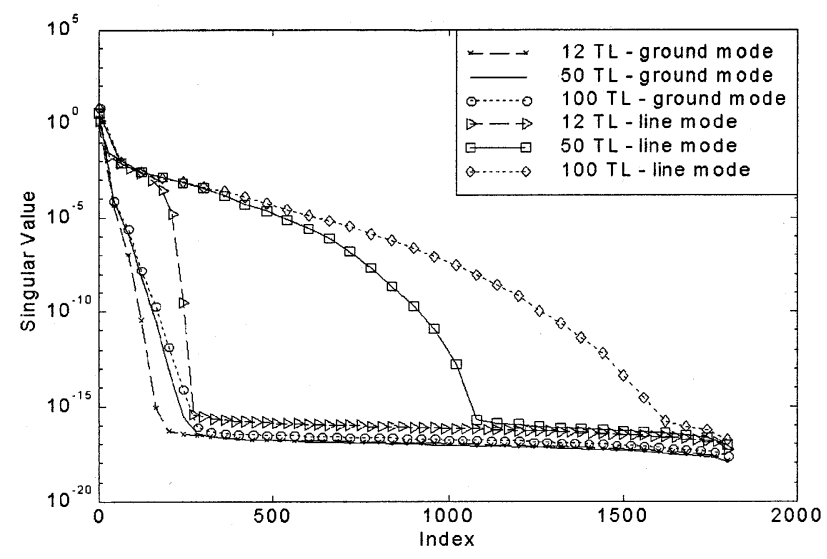

Fig. 1. Singular value decomposition of $C_{L}$ for networks composed of 12, 50, and 100 transmission lines.

1.5. Usually, (3) is poorly conditioned when the ground mode is considered. An example is shown in Fig. 1, where the SVDs of $C_{L}$ corresponding to both modes for systems composed of 12,50 , and 100 transmission lines are presented. The parameters for these calculations are $N=4000, p=1800$, and the time step is $50 \mu \mathrm{s}$. The determined values for $p$ (line mode) are 242,916 , and 1262 , respectively, for the 12,50 , and 100 transmission lines systems. These results show that even a very large system, for instance 100 transmission lines, can be represented by an equivalent using a relatively small value for $p$.

\section{B. Sparsity in the Identification of $a_{k}$ and $b_{k}$}

Sparsity means zero values in the coefficients $a_{k}, b_{k}$. It has the beneficial effect of reducing the time needed for the calculation of the convolutions in (5) and correspondingly speeding up the computation of transients. Enforcing sparsity in the sets $a_{k}$ and $b_{k}$ implies to identify which coefficients will have nonzero values while the others will be set to zero. This is accomplished by obtaining a basic solution of (3) using methods based on QR factorization and searching the solution for the position of the $N_{S}$ largest coefficients for each set $a_{k}$ and $b_{k}$. These positions are saved and used in latter calculations as described in Section V. This approach is suitable for obtaining equivalents for both line and ground modes. The use of QR factorization (achieved by the " $\backslash$ " operator in Matlab [21]) allows one to take advantage of the rank deficiency typical for the ground mode by automatically setting to zero a number of variables. This results in an initial sparsity in the basic solution of (3). The same could be obtained using SVD, but at a higher computational cost. The details of how this initial sparsity is obtained using SVD or QR factorization are presented in Appendix A. An alternative procedure for the line mode is presented in the following. It is based on the analysis of the current sequence $i(n)$.

When $i(n)$ is calculated for a small system, the reflected traveling waves arrive at the sending bus separated by relatively large time intervals. The comparison between the positions of the nonzero coefficients obtained from a basic solution of (3) and the instants at which traveling waves arrive at the sending bus, shows a great degree of coincidence. For a large system, such a characteristic is not prominent due to the increased number of traveling waves arriving at the sending bus. This 
causes the basic solution of (3) to be practically nonsparse, making the identification of the best positions for the nonzero coefficients difficult. It is, however, possible to obtain the positions for the nonzero coefficients which reflect the arriving waves by analyzing $i(n)$ as follows.

First, we calculate a pseudo impulse response $i_{I R}(n)$ as the backward difference of $i(n), i_{I R}(n)=i(n)-i(n-1)$. The largest values of $i_{I R}(n)$, in magnitude, correspond to the arrival instants of the traveling waves. A search for the $N_{S}$ largest values of $i_{I R}(n)$, for $n=0, \ldots, p-1$, identifies the positions of the nonzero coefficients for the set $a_{k}$. For the set $b_{k}$, the positions are obtained from a basic solution of (3) with $C$ built only for the nonzero unknowns already identified. The part of the basic solution corresponding to $b_{k}$ is searched and the positions of the $N_{S}$ largest elements are identified.

In calculations corresponding to enforcement of stability, passivity, and accuracy for specific frequencies, only the nonzero unknowns are used.

\section{CONSTRAINT EQUATIONS}

The sparsity of the sets $a_{k}$ and $b_{k}$ results in significant computational efficiency for the equivalent. However, it usually produces an unstable, nonpassive equivalent.

The constraint equations derived in this section are used to build $D$ and $e$ for (1). The stability and passivity of a linear system is related to the coefficients $a_{k}$ and $b_{k}$ through nonlinear equations. These constraints are enforced using a linearized form of such equations in an iterative procedure. Although transient calculations using the equivalents can provide a good overall fitting, the equivalent itself may lack accuracy at specific frequencies like 0 and $60 \mathrm{~Hz}$. Thus, single frequency constraints are used to improve the accuracy at such frequencies. It is important to mention that forcing a high accuracy at special frequencies does not imply large penalties for the overall fitting error, as the results presented in the companion paper demonstrate.

\section{A. Stability Constraint}

The information regarding the stability of a linear system, described by a constant-coefficients difference equation, is assessed through the analysis of its natural modes. This is done by calculating its zero-input response, that is, the sequence $i_{h}(n)$ that satisfies

$$
\sum_{k=0}^{p} a_{k} i_{h}(n-k)=0 .
$$

A solution for (6), referred to as the homogeneous equation [22], is a sequence $i_{h}(n)$ of the form

$$
i_{h}(n)=\text { const. } z^{n} \text {. }
$$

From the substitution of (7) in (6), the complex numbers $z$ in $i_{h}(n)$ must be the roots of the polynomial equation

$$
\sum_{k=0}^{p} a_{k} z^{-k}=0
$$

The absolute value for each root $z$ must be less than 1 to result in a stable sequence. Suppose we have a basic solution for
(1) that results in an unstable equivalent. Assuming linearity, to enforce stability, we calculate a correction $\Delta x$ to be added to $x$ such that the absolute values of all roots $z$ become less than 1 . The stability constraint equation is then

$$
J_{S} \Delta x<1-\left|z_{U}\right|
$$

where $J_{S}$ is the Jacobian matrix of the absolute values of $z_{U}$, the unstable (and potentially unstable) set of roots, with respect to the coefficients $a_{k}$. The elements of $J_{S}$ can be calculated analytically using

$$
\frac{\partial\left|z_{j}\right|}{\partial a_{i}}=\left|z_{j}\right| \operatorname{Re} \frac{1}{\sum_{k=1}^{p} k a_{k} z_{j}^{-k+i}} .
$$

The details for deriving (10) are given in Appendix B. The constraint for stability is expressed as a function of the increment $\Delta x$ and the corresponding incremental formulation of (1) is given in Section $\mathrm{V}$.

\section{B. Passivity Constraint}

The equivalent represents the admittance of the external system and, like every passive network, when represented as a complex valued function in the frequency domain, it must have its real part positive.

The external system is excited by a voltage source

$$
v(n)=v\left(t_{n}\right)=v(n \Delta t)=v_{0} e^{j \omega n \Delta t}=v_{0}\left(e^{j \omega \Delta t}\right)^{n} .
$$

Using the auxiliary variable $z=e^{j \omega \Delta t},(11)$ is expressed as

$$
v(n)=v_{0} z^{n} \text {. }
$$

The corresponding current is

$$
i(n)=i_{0} z^{n} \text {. }
$$

Setting $v_{0}=1$, which implies $i_{0}=Y_{\omega}$, and substituting (11) and (12) in (5), we obtain

$$
Y_{\omega}=b_{0}+\sum_{k=1}^{p}\left(b_{k}-Y_{\omega} a_{k}\right) z^{-k} .
$$

Equation (14) is a representation of the equivalent as an admittance and a function of $\omega$. Rearranging (14) leads to

$$
Y_{\omega}=\frac{\sum_{k=0}^{p} b_{k} z^{-k}}{1+\sum_{k=1}^{p} a_{k} z^{-k}}=G_{\omega}+j B_{\omega}=\frac{N_{\omega}}{D_{\omega}} .
$$

From a basic solution of $x$, we compute $Y_{\omega}$ for several frequencies resulting in the set $Y_{0}$ for which the real part is $G_{0}$. We need to calculate two corrections $\Delta x_{a}$ and $\Delta x_{b}$ (for $a_{k}$ and $b_{k}$, respectively) to be added to $x$ in order to enforce the value of $G_{0}$ to be positive for any $\omega$. Thus, the passivity constraint equation is

$$
\left[\begin{array}{ll}
-J_{P a} & -J_{P b}
\end{array}\right]\left[\begin{array}{c}
\Delta x_{a} \\
\Delta x_{b}
\end{array}\right]<G_{0}
$$

where $J_{P a}$ and $J_{P b}$ are the Jacobian matrices of $G_{0}$ with respect to the coefficients $a_{k}$ and $b_{k}$, respectively. The components of $J_{P a}$ and $J_{P b}$ are calculated using (17) and (18), respectively, derived directly from (15)

$$
\begin{aligned}
& \frac{\partial G_{\omega}}{\partial a_{k}}=-\operatorname{Re} \frac{N_{\omega}}{D_{\omega}^{2}} e^{-j \omega k} \\
& \frac{\partial G_{\omega}}{\partial b_{k}}=\operatorname{Re} \frac{e^{-j \omega k}}{D_{\omega}}
\end{aligned}
$$




\section{Single-Frequency Accuracy Constraint}

Once the complex value $Y_{\omega_{o}}=G_{\omega_{o}}+j B_{\omega_{o}}$ of the admittance of the external system is known for a particular frequency $\omega_{o}$, it can be used to enforce the required accuracy of the equivalent at this specific frequency. The equations for this constraint are similar to (16) but are equality constraints and also deal with the imaginary part of $Y_{\omega_{o}}$

$$
\begin{aligned}
& {\left[\begin{array}{ll}
J_{r P a_{o}} & J_{r P b_{o}}
\end{array}\right]\left[\begin{array}{l}
\Delta x_{a} \\
\Delta x_{b}
\end{array}\right]=G_{\omega_{o}}-G_{o o}} \\
& {\left[\begin{array}{ll}
J_{i P a_{o}} & J_{i P b_{o}}
\end{array}\right]\left[\begin{array}{l}
\Delta x_{a} \\
\Delta x_{b}
\end{array}\right]=B_{\omega_{o}}-B_{o o} .}
\end{aligned}
$$

In (19) and (20), the right side represents mismatches. $G_{o o}$ and $B_{o o}$ are, respectively, the real and imaginary parts of $Y_{\omega}$, calculated at $\omega_{o}$, from the basic solution $x$ and $J_{i P a_{o}}\left(J_{r P a_{o}}\right)$ and $J_{i P b_{o}}\left(J_{r P b_{o}}\right)$ are Jacobian obtained from the imaginary (real) counterpart of (17) and (18), computed only at $\omega_{o}$. Each single frequency admittance value that is to be enforced, needs a pair of equations like (19) and (20), except for $0 \mathrm{~Hz}$ where only (19) is used.

\section{STEP-By-STEP PROCEDURE FOR NeTWORK EQUiVALENT CALCUlation}

The fitting and constraint equations derived in Sections III and IV are combined in an iterative procedure that uses quadratic programming to obtain a solution for (1) in each iteration. The procedure must be iterative due to the intrinsically nonlinear relation between the constraints and the unknowns, the sets $a_{k}$ and $b_{k}$. The equivalents for both line and ground modes are calculated separately using the procedure outlined in the following.

Step 1) Using SVD and the "length" of the network (based on the concepts in Section III-A), determine a suitable value for $p$.

Step 2) From (3), build matrices $C$ and $V$ from data sequences $i(n)$ and $v(n)$ using $q=p$.

Step 3) Following the procedure presented in Section III-B, identify positions of $N_{S}$ nonzero coefficients for each set $a_{k}$ and $b_{k}$. If the equivalent is calculated for the line mode, the pseudo impulse response procedure may be used. Our studies show that this procedure usually results in a better overall fitting for the same degree of sparsity.

Step 4) Modify matrices $C$ and $V$ keeping only the columns corresponding to the $N_{S}$ nonzero coefficients for each set $a_{k}$ and $b_{k}$. The corresponding sparse version of matrix $A$, in (1), is called $A_{S p}$. Obtain a basic sparse solution $x_{S p}$ and split it in the corresponding sparse sets of coefficients $a_{k}$ and $b_{k}$.

Step 5) Using (8), calculate the set of roots $z_{U}$. From (15), obtain $G_{0}$.

Step 6) The iterative procedure begins at this step. Calculate the Jacobians $J_{r P a_{o}}, J_{r P b_{o}}, J_{i P a_{o}}$, and $J_{i P b_{o}}$, at each single frequency $\omega_{o}$ using $Y_{\omega_{o}}$.

Step 7) To reduce the computational effort in the quadratic programming procedure, only parts of the sets $z_{U}$ and $G_{0}$ are used. From $z_{U}$, only the roots with absolute values larger or slightly less than 1 are used. Similarly, from $G_{0}$, we use only those frequencies at which $G_{\omega}$ is negative or slightly positive. Calculate Jacobians matrices $J_{S}, J_{P a}$, and $J_{P b}$ for the stability and passivity enforcement only for the roots and frequencies specified above.

Step 8) Combine the constraint equations to build $D$ and $e$. Step 9) As the constraint equations are expressed in terms of increments for the unknowns, the corresponding version of (1), shown in (21), is used in the quadratic programming procedure

$$
\min _{\Delta x_{S p}}\left\|A_{S p} \Delta x_{S p}-\left(c-A_{S p} x_{S p}\right)\right\|, \quad D \Delta x_{S p} \leq e .
$$

Step 10) Using $\Delta x_{S p}$ from the last step, calculate the solution $x_{S p}+\Delta x_{S p}$. Split it in the sets $a_{k}$ and $b_{k}$. Calculate $z_{U}$ and $G_{0}$. Check if the single frequency accuracy, stability, and passivity requirements are satisfied. If all of the requirements are met, terminate the iterative procedure. If not, go back to Step 6 and continue the iterations until all of the requirements are met.

The speed of convergence of the iterative procedure can be of concern. From several calculations of equivalents, we observed that the procedure assures robustness in about 5-15 iterations.

\section{CONCLUSIONS}

The paper presents a methodology for the calculation of a robust SNE based on time-domain fitting. The methodology allows obtaining equivalents for very large systems. The SNE can be directly integrated in an electromagnetic transient calculation program. Its sparsity assures computational efficiency.

The robustness of the SNE is accomplished by enforcing a set of linear constraints, accounting for stability, passivity, and accuracy at specific frequencies. The equivalent is calculated by an iterative procedure as a constrained least squares solution of the fitting equations using a quadratic programming procedure.

The results presented in the companion paper work demonstrate the accuracy and computational efficiency of the new network equivalent. It is shown that, due to its sparsity, the SNE is by an order of magnitude faster than a nonsparse equivalent.

\section{APPENDIX A}

\section{SPARSITY IN THE BASIC SOLUTION OF (3) USING SVD OR QR FACTORIZATION}

The matrix factorizations of SVD and QR can be used to obtain a basic solution for (3) which ab initio has some degree of sparsity. The sparsity corresponds to the rank deficiency of $A$, in (1), and is more pronounced in the case of the ground-mode equivalent. For more general derivations, see [25]. We address the use of SVD first.

The basic solution of (3) is obtained solving, in a least-squares sense

$$
A x=c
$$


where $A=[C-V], A \in \mathbb{R}^{n \times m}$. Taking the SVD of $A$ we obtain

$$
A=U \Sigma V^{T} \text {. }
$$

If the rank of $A$ is $r, r<m$, the diagonal matrix $\Sigma \in \mathbb{R}^{n \times m}$, can be partitioned as follows:

$$
\Sigma=\left[\begin{array}{cc}
\sum_{1} & 0 \\
0 & \sum_{2}
\end{array}\right]
$$

where the submatrix $\Sigma_{1} \in \mathbb{R}^{r \times r}$ contains the significant singular values of $A$ and $\Sigma_{2} \in \mathbb{R}^{n-r \times m-r}$, the very small singular values. Substituting (A.2) in (A.1) we obtain

$$
\Sigma y=g
$$

where

$$
\begin{aligned}
& g=U^{T} c, \quad c=U g, \\
& y=V^{T} x, \quad x=V y .
\end{aligned}
$$

Neglecting $\Sigma_{2}$ and solving (A4) in the partitioned form

$$
\left[\begin{array}{cc}
\sum_{1} & 0 \\
0 & 0
\end{array}\right]\left[\begin{array}{l}
y_{1} \\
y_{2}
\end{array}\right]=\left[\begin{array}{l}
g_{1} \\
g_{2}
\end{array}\right]
$$

we observe that $y_{2}$ can be chosen arbitrarily and the lower part of (A.7) is satisfied only if $g_{2}$ is zero, implying a final (small) error in the least-squares solution. We get $y_{1}=\left(\Sigma_{1}\right)^{-1} g_{1}$.

If we write $x=V_{y}$ of (A.6) in the partitioned form

$$
\left[\begin{array}{l}
x_{1} \\
x_{2}
\end{array}\right]=\left[\begin{array}{ll}
V_{11} & V_{12} \\
V_{21} & V_{22}
\end{array}\right]\left[\begin{array}{l}
y_{1} \\
y_{2}
\end{array}\right]
$$

we see that the arbitrary $y_{2}$ can be chosen so as to set part of $x$ to zero, resulting in the desired sparsity. These restrictions to be imposed on $x$ can be formulated as

$$
L x=h
$$

where $h \in \mathbb{R}^{m \times 1}$ is all zeros and $L \in \mathbb{R}^{m-r \times m}$ is all zeros except in the corresponding positions of the part of $x$ to be set to zero. Premultiplying (A.8) by $L$ and taking (A.9) and (A.6) into account, results in

$$
\left[\begin{array}{ll}
W_{1} & W_{2}
\end{array}\right]\left[\begin{array}{l}
y_{1} \\
y_{2}
\end{array}\right]=h, \quad W=L V .
$$

Solving (A.10), that is

$$
W_{2} y_{2}=h-W_{1} y_{1}
$$

for $y_{2}$ we can obtain, using (A6), the corresponding $x$, with $m-r$ variables equal to zero, resulting in the desired sparsity.

There is still the problem of which values of $x$ are to be set to zero. Obviously, these should be the smallest in a nonsparse solution. This information may come from the permutation matrix obtained in the $\mathrm{QR}$ factorization of $A$. Indeed, QR not only provides such information but also automatically results in the sparse solution for (A.1). The difference between SVD and QR is mainly in the norm of the obtained solution; however, both approaches give similar residues for (A.1). SVD results in the solution with the lower norm.

If the QR factorization with column pivoting is used in (A.1), we have

$$
A=Q R P^{T}, \quad Q R P^{T} x=c
$$

where $P \in \mathbb{R}^{m \times m}$ is a permutation matrix and $R \in \mathbb{R}^{n \times m}$ is upper triangular and is partitioned as

$$
R=\left[\begin{array}{cc}
R_{11} & R_{12} \\
0 & R_{22}
\end{array}\right] .
$$

In (A.13), $R_{11} \in \mathbb{R}^{r \times r}$ is full rank and $R_{22} \in \mathbb{R}^{n-r \times m-r}$ has only negligible elements resulting from the ill-conditioning of $A$. Using a procedure similar to that for the SVD approach, we solve for $y_{1}$ as $R_{11} y_{1}=g_{1}$, with $g=Q^{T} c$ and $y=P^{T} x$. Now, the sparse solution for $x$ is obtained simply from

$$
P\left[\begin{array}{c}
y_{1} \\
0
\end{array}\right]=x \text {. }
$$

QR factorization with pivoting is known to be reliable in determining the most linearly independent set of columns of a rank deficient matrix $A$, and linking it to $R_{11}$ by means of the permutation matrix $P$. SVD is less favored due to its high computational cost. Linear algebra packages usually provide operators for least-squares solutions, like the "\" operator in Matlab [21], that automatically set to zero a number of variables equal to the rank deficiency of the $A$ matrix.

\section{APPENDIX B}

\section{DERIVATION OF THE ANALYTICAL FORMULA FOR $\partial\left|z_{j}\right| / \partial a_{i}$}

From the polynomial (8), which governs the stability of the equivalent, we obtain a formula for derivatives of the absolute value of each root of (8), $\left|z_{j}\right|$, with respect to each coefficient of the set $a_{k}$. These derivatives could be calculated numerically but at a much higher computational cost and less accuracy. In a general formulation, the roots of (8) must satisfy the following multivariable function:

$$
f\left(z, a_{0}, a_{1}, a_{2}, \ldots, a_{i}, \ldots, a_{p}\right)=\sum_{k=1}^{p} a_{k} z^{-k}=0 .
$$

Taking the total differential of $f$, in the vicinity of the root $z=$ $z_{j}$, and assuming that only $a_{i}$ varies results in

$$
\frac{\partial z_{j}}{\partial a_{i}}=-\frac{\left.\frac{\partial f}{\partial a_{i}}\right|_{z=z_{j}}}{\left.\frac{\partial f}{\partial z}\right|_{z=z_{j}}}=-\frac{z_{j}^{-i}}{\sum_{k=1}^{p}-k a_{k} z_{j}^{-k-1}} .
$$

Therefore

$$
\frac{\partial\left|z_{j}\right|}{\partial a_{i}}=\lim _{\Delta a_{i} \rightarrow 0} \frac{\left|z_{j}+\frac{\partial z_{j}}{\partial a_{i}} \Delta a_{i}\right|-\left|z_{j}\right|}{\Delta a_{i}}=\left|z_{j}\right| \operatorname{Re} \frac{1}{z_{j}} \frac{\partial z_{j}}{\partial a_{i}} .
$$

Taking (B.2) and (B.1) into account, we obtain (10) as

$$
\frac{\partial\left|z_{j}\right|}{\partial a_{i}}=\left|z_{j}\right| \operatorname{Re} \frac{z_{j}^{-i-1}}{\sum_{k=1}^{p} k a_{k} z_{j}^{-k-1}}=\left|z_{j}\right| \operatorname{Re} \frac{1}{\sum_{k=1}^{p} k a_{k} z_{j}^{-k+i}} \text {. }
$$

\section{ACKNOWLEDGMENT}

The first author wishes to thank CAPES/Brazil and the Federal University of Minas Gerais for granting a study leave at the University of Toronto.

\section{REFERENCES}

[1] A. S. Morched and V. Brandwajn, "Transmission network equivalents for electromagnetic transient studies," IEEE Trans. Power App. Syst., vol. PAS-102, pp. 2984-2994, Sept. 1983. 
[2] A. S. Morched, J. H. Ottevangers, and L. Marti, "Multi-port frequency dependent network equivalents for the EMTP," IEEE Trans. Power Delivery, vol. 8, pp. 1402-1412, July 1993.

[3] V. Q. Do and M. M. Gavrilovic, "An iterative pole-removal method for synthesis of power system equivalent networks," IEEE Trans. Power App. Syst., vol. PAS-103, pp. 2065-2070, Aug. 1984.

[4] , "A synthesis method for one-port and multi-port equivalents network for analysis of power system transients," IEEE Trans. Power Syst., vol. PWRD-1, pp. 103-113, Apr. 1986.

[5] A. Semlyen and M. R. Iravani, "Frequency domain modeling of external systems in an electro-magnetic transients program," IEEE Trans. Power Syst., vol. 8, pp. 527-533, May 1993.

[6] J.-H. Hong and J.-K. Park, "A time-domain approach to transmission network equivalents via prony analysis for electromagnetic transients analysis," IEEE Trans. Power Syst., vol. 10, pp. 1789-1797, Nov. 1995.

[7] H. Okamoto, A. Kurita, J. J. Sanchez-Gasca, K. Clark, N. W. Miller, and J. H. Chow, "Identification of equivalent linear power system models from electromagnetic transient time domain simulations using Prony's method," in Proc. 35th Conf. Decision Contr., Kobe, Japan, Dec. 1996, pp. $3857-3863$

[8] — "Identification of low order linear power system models from EMTP simulations using Steiglitz-McBride algorithm," IEEE Trans. Power Syst., vol. 13, pp. 422-427, May 1998.

[9] J. R. Smith, F. Fatehi, C. S. Woods, J. F. Hauer, and D. J. Trudnowski, "Transfer function identification in power system applications," IEEE Trans. Power Syst., vol. 8, pp. 1282-1290, May 1993.

[10] A. Abur and H. Singh, "Time domain modeling of external systems for electromagnetic transients programs," IEEE Trans. Power Syst., vol. 8, pp. 671-679, May 1993.

[11] H. Singh and A. Abur, "Multi-port equivalencing of external systems for simulation of switching transients," IEEE Trans. Power Delivery, vol. 10, pp. 374-382, Jan. 1995.

[12] T. Noda, N. Nagaoka, and A. Ametani, "Phase domain modeling of frequency-dependent transmission lines be means of an ARMA model," IEEE Trans. Power Delivery, vol. 11, pp. 401-411, Jan. 1996.

[13] J. J. Sanchez-Gasca and J. H. Chow, "Performance comparison of three identification methods for the analysis of electromechanical oscillations," IEEE Trans. Power Syst., vol. 14, pp. 995-1002, Aug. 1999.

[14] J. R. Marti, "Accurate modeling of frequency-dependent transmission lines in electromagnetic transient simulations," IEEE Trans. Power App. Syst., vol. PAS-101, pp. 147-155, Jan. 1982.

[15] J. Lin and J. R. Marti, "Implementation of the CDA procedure in the EMTP," IEEE Trans. Power Syst., vol. 5, pp. 394-402, May 1990.

[16] A. Semlyen and F. de Leon, "Computational of electromagnetic transients using dual or multiple time steps," IEEE Trans. Power Syst., vol. 8, pp. 1274-1281, Aug. 1992

[17] G. Angelidis and A. Semlyen, "Direct phase-domain calculation of transmission line transients using two-sided recursions," IEEE Trans. Power Delivery, vol. 10, pp. 941-949, Apr. 1995.

[18] T. Henriksen, "Including high order rational functions in EMTP a comparison between alternative methods with emphasis on accuracy," IEEE Trans. Power Delivery, vol. 12, pp. 372-379, Jan. 1997.

[19] T. Noda, N. Nagaoka, and A. Ametani, "Further improvements to a phase-domain ARMA line model in terms of convolution, steady-state initialization, and stability," IEEE Trans. Power Delivery, vol. 12, pp. 1327-1334, July 1997.

[20] H. V. Nguyen, H. W. Dommel, and J. R. Marti, "Direct phase-domain modeling of frequency-dependent OVERHEAD transmission lines," IEEE Trans. Power Delivery, vol. 12, pp. 1335-1342, July 1997.
[21] Matlab-Language Reference Manual. Natick, MA: The MathWorks, Inc., June 1997, vol. 5.

[22] A. V. Oppenheim and R. W. Schafer, Discrete-Time Signal Processing. Englewood Cliffs, NJ: Prentice-Hall, 1989.

[23] R. Prony, "Essai expérimental et analytique sur les lois de la dilatabilité des fluides élastiques et sur celles de la force expansive de la vapeur d'alcool à différentes températures," in J.l'Ecole Polytech. Paris, France, 1795, vol. 1, pp. 24-76.

[24] M. L. Van Blaricum and R. Mittra, "Problems and solutions associated with Prony's method for processing transient data," IEEE Trans. Antennas Propagat., vol. AP-16, pp. 174-182, Jan. 1978.

[25] G. H. Golub and C. F. Van Loan, Matrix Computations, 2nd ed. Baltimore, MD: The John Hopkins Univ. Press, 1989.

Wallace do Couto Boaventura (M'94) was born in 1965 in Brazil. He received the B.Sc. and M.Sc. degrees from the Federal University of Minas Gerais, Belo Horizonte, Brazil, in 1988 and 1990, respectively. He is currently pursuing the $\mathrm{Ph} . \mathrm{D}$. degree in signal processing applications in power systems at the University of Campinas, Campinas, Brazil/University of Toronto, Toronto, ON, Canada.

Currently, he is an Assistant Professor at the Federal University of Minas Gerais, where he has been since 1992 .

Adam Semlyen (LF'97) was born in 1923 in Rumania. He received the Dipl Ing. degree from the Polytechnic Institute of Timisoara, Timisoara, Romania, in 1950, and the Ph.D. degree from the Polytechnic Institute of Iasi, Iasi, Romania, in 1965 .

Since 1988, he has been Professor Emeritus in the Department of Electrical and Computer Engineering, Univeristy of Toronto, Toronto, ON, Canada, where he has been since 1969. His research interests include steady-state and dynamic analysis as well as computation of electromagnetic transients in power systems.

M. Reza Iravani (SM'00-F'03) received the B.Sc. degree in electrical engineering from Tehran Polytechnic University, Tehran, Iran, in 1976, and the M.A.Sc. and Ph.D. degrees in electrical engineering from the University of Manitoba, Winnipeg, MB, Canada, in 1981 and 1985, respectively.

Currently, he is a Professor at the University of Toronto, Toronto, ON, Canada. His research interests include power system transients and power electronics.

Amauri Lopes (M'80) received the B.Sc., M.Sc., and Ph.D. degrees in electrical engineering from the University of Campinas, Campinas, Brazil, in 1972, 1974, and 1982 , respectively.

Currently, he is an Associate Professor with the Electrical and Computer Engineering School at the University of Campinas. His research interests include digital signal processing and wireless communications. 